\title{
DIE THEORETISCHEN GRUNDLAGEN DER AKTION LASSALLES IM BRIEFWECHSEL MIT RODBERTUS
}

Der Briefwechsel Rodbertus-Lassalle umfasst die ganze Zeit der Arbeiteragitation. Er beginnt mit dem 25. Dezember I 862, nachdem Lassalle schon mit dem Komitee der Leipziger in Verbindung getreten war, und endigt erst kurz vor seinem Tode mit einem Brief vom I4. Juni I 864 .

In diesem Zeitabschnitt ist Lassalle sehr darauf bedacht, sich in allen öffentlichen Äusserungen keine Blösse zu geben, die gegen ihn agitatorisch ausgenützt werden könnte. Daher weckt er den Schein der Inkongruenz zwischen dem theoretisch-revolutionären „System der erworbenen Rechte", das die Basis der dreijährigen demokratischrevolutionären Propaganda war, und der streng legalistischen Arbeiteragitation der letzten beiden Lebensjahre. ${ }^{1}$ Der Briefwechsel mit Rodbertus ist insofern von besonderer Bedeutung, als schon die durch Rodbertus gestellten Themen einer agitatorischen Ausnützung der Korrespondenz entgegenwirkten. Allein die Tatsache, dass Lassalle zur Zeit der Arbeiteragitation mit Rodbertus die weitgehendsten Konsequenzen seines „Systems” erörtert, ist von Bedeutung, da

1 „Unter dem Namen 'Allgemeiner Deutscher Arbeiterverein' begründen die Unterzeichneten für die deutschen Bundesstaaten einen Verein, welcher... den Zweck verfolgt auf friedlichem und legalem Wege... für die Herstellung des allgemeinen, gleichen und direkten Wahlrechts zu wirken". (Ferdinand Lassalle, Gesammelte Reden und Schriften, hrsg. und eingel. von Eduard Bernstein. Cassirer, Berlin 1919, Bd. 4. S. 246; wobei das Wort „Herstellumg” auf preussische Verfassungsverhältnisse bezogen sehr verfänglich ist.) Über die revolutionären Konsequenzen des „Systems der erworbenen Rechte” am klarsten: F. A. Lange, Die Arbeiterfrage, 3. Aufl. Winterthur 1875: „Das System der entzogenen Rechte” - „Das Lassallesche Buch ist ihnen aber unheimlich, da sie wohl fühlen, dass das eigentliche Fazit des Buches erst hinter der letzten Zeile kommt”. S. 399-400. 
dadurch die rein realpolitisch-opportunistische Interpretation von Lassalles sozialer Aktion hinfällig wird.

Rodbertus ist der einzige, der Lassalle herausgefordert hat, die tieferen Grundlagen seines Systems zu erörtern und damit vor sich selbst in Frage zu stellen. Lassalle sollte ihm sein Gesellschaftsideal und seinen Begriff der dialektischen Einheit darstellen und sie verteidigen. Die Art, in der Lassalle auf diese Herausforderung reagiert hat, gibt genau den Standort seiner Theorie zur Zeit der Arbeiteragitation an. Selbst der jahrelange Briefwechsel von Lassalle mit Marx und Engels ist in dieser Beziehung nicht so bedeutsam wie der vorliegende, denn zwischen Marx-Engels und Lassalle bestand eine Art von stillschweigender Übereinkunft, nicht die philosophischen, sondern nur die politischen Voraussetzungen ihrer Meinungsverschiedenheiten zu berühren.

Der Briefwechsel gibt aber nicht nur Aufschluss über die tieferen Gründe und letzten Ziele der Arbeiterpolitik; er spiegelt auch die taktische Lage Lassalles wider, der Rodbertus braucht, um sein Konzept von der Einheit der „Wissenschaft” und der „Arbeiter” zu verwirklichen. Wenn er auch nie versucht hat, seinen Briefwechsel mit Rodbertus agitatorisch auszubeuten, wie er es etwa den mit Huber geführten getan hat, so darf man doch nie aus den Augen verlieren, dass Lassalle bei Rodbertus etwas Bestimmtes erreichen will.

Desgleichen will auch Rodbertus etwas bei Lassalle erreichen. Er erkennt in Lassalles straff organisierter Bewegung sowohl die Möglichkeiten zu einer grossen sozialen Bewegung, die auch ihm festen Boden unter die Füsse geben kann, wie auch die Gefahr politischer Entgleisung, die dem Cäsarismus die Wege ebnet. ${ }^{1}$ Am wenigsten möchte er für immer der "Theoretiker von Jagetzow” bleiben. ${ }^{2}$ Wir beachten darum auch Rodbertus' Äusserungen unter taktischen Gesichtspunkten.

\section{II}

Rodbertus' Briefe sind durchdacht und ausführlich und darum eine unerlässliche Ergänzung der Lassalleschen, da erst durch sie sowohl die Möglichkeiten wie die Grenzen von Lassalles Denken erhellt werden.

1 Bd. 6, S. 343. Rodbertus sah mit Enttäuschung, wie wenig Wirkung seine mit Bucher veröffentlichten Flugblätter hatten. „Die Reinheit der sozialen Frage erscheint immer in Gegenüberstellung zum politisch-revolutionären Weg", wobei dem Scheitern des revolutionären Weges die Hauptsorge gilt. S. 343 .

2 So mehrfach G. Mayer. z.B. Einleitung Bd. 6. S. 291. 
Der Briefwechsel enthält wahre Abhandlungen, und spiegelt zugeleich das Bild einer lebhaften Diskussion wider. Sowohl Lassalle wie auch Rodbertus erwarten von der theoretischen Auseinandersetzung praktische Resultate in Hinblick auf den Arbeiterverein; und der Briefwechsel ist gerade in dieser Hinsicht eine bedeutsame Dokumentation.

Die ersten vier Briefe behandeln Fragen, die noch an das „System der erworbenen Rechte" anknüpfen. Rodbertus greift Lassalles Ausgangspunkt vom freien Willen des Individuums her an und entwickelt die Grundzüge einer organischen Gesellschafts- und Staatsauffassung. ${ }^{1}$ Damit schafft er die Grundlagen zu einer Auseinandersetzung mit Lassalles Assoziationsplänen, die durch die Veröffentlichung des „Offenen Antwortschreibens" aktuell geworden sind.

Die folgenden $2 x$ Briefe stehen im Zeichen des Ringens um Inhalt und Form der Arbeiterbewegung und erreichen ihren Höhepunkt am Vorabend der entscheidenden Versammlung zu Frankfurt, die für Lassalle positiv, für Rodbertus negativ ausgeht. Inhalt und Taktik der Bewegung machen Rodbertus' aktive Beteiligung unmöglich; die Differenzen werden schärfer herausgearbeitet und gipfeln in der Kontroverse: Staatsbetrieb mit Arbeitslobn oder Assoziationen mit Gewinnanteil. Rodbertus' Ausgangspunkt vom Gemeinwesen, wie er im ersten Teil des Briefwechsels dargestellt wurde, wird nun mit Lassalles Ausgangspunkt vom Individuum konfrontiert. ${ }^{2}$ In sechswöchigem intensivem Ringen werden Grundlagen und letzte Ziele der Bewegung durchgekämpft.

Die letzten 17 Briefe schreibt man in weit grösserem Abstand voneinander. ${ }^{3}$ Sie sind hauptsächlich Nebenprodukte des „BastiatSchulze" und werden auch so von Rodbertus gewürdigt. Die Entscheidung, welchen Weg die Arbeiterbewegung zu gehen habe, ist gegen Rodbertus gefallen und die tagtägliche Entwicklung der Bewegung erleichtert ein stärkeres Betonen der Gegensätze.

Die Tatsache, dass Lassalle in den Fragen der Bodenrente bei Ricardo stehen geblieben ist, war sicher eine grosse Enttäuschung für Rodbertus, der gerade in seiner Rententheorie sein Hauptverdienst sah.

Die späteren Äusserungen von Lothar Bucher und Adolf Wagner in Rodbertus' Namen betonen die besonderen Differenzen. Die Abhängigkeit Lassalles von Proudhon wie auch die Schichtung in einen

1 Bd. 6. S.297-321.

2 Bd. 6. S. $321-260$.

3 Bd. 6. S. 360-381. 
„exoterischen” und „esoterischen” Lassalle wird ganz anders dargestellt als sich aus der Korrespondenz ergibt, die dadurch auch Rodbertus gegenüber ein Korrektiv ist, denn gerade er hat diese Schichtung gefordert, und Lassalle ist nur sein Widerhall. ${ }^{1}$ Oncken hat viel psychologischen Scharfsinn auf die Deutung der sich daraus ergebenden Charakterzüge verwendet.

Lassalle knüpft die Beziehungen zu Rodbertus schon im Hinblick auf die kommende Arbeiteragitation an. Er verweist Rodbertus besonders auf den $\$ 7$ seines „Systems”, der den systematischen Übergang von demokratischer zu sozialdemokratischer Aktion ermöglicht.

Am Ende der ersten Phase des Briefwechsels erscheint das „Offene Antwortschreiben”, in dem Rodbertus mit Recht eine von Lassalles "System" unabhängige Grundlage für die soziale Agitation feststellt: Der Übergang von der demokratischen zur sozialdemokratischen Aktion erweist sich so als Krise der Theorie Lassalles.

In seinem „System” hatte Lassalle vom Standpunkt der formalen Rechtslogik aus ein System geordneter Änderung der Eigentumsverhältnisse geschaffen; er hatte die Revolution der Rechtsverhältnisse in ein Rechtssystem eingebaut, die Revolution zur Rechtsnorm erhoben, sofern sie sich an gewisse formale Regeln hält. Lassalle glaubte mit Recht, dass Rodbertus diese Theorie übernehmen würde. Rodbertus war sogar bereit, noch viel weiter zu gehen: Für ihn war der Verletzung individuellen Willens bei rückwirkender Kraft neuer Gesetzgebung keine absolute Schranke gesetzt. ${ }^{2}$

Lassalle geht bei seiner Darlegung vom abstrakt persönlichen und willkürlichen Willen aus, der bei zunehmender Konkretisierung sich immer mehr in den Allgemeinwillen einbaut, aber formal immer Einzelwille bleibt und als solcher geachtet werden muss. Rodbertus kennt keinen von der organischen Einheit losgelösten Einzelwillen. Es ist ihm Ernst mit dem Bild von den Zellen, die erst durch den Staatskörper lebensfähig werden. Für ihn ist der Staat „frei" und seinem Willen, Unrecht gut zu machen, sind keine Schranken gesetzt. ${ }^{3}$

1 Brief Nr. II.

2 Brief Nr. 2, S. 302-3. ,je mehr aber endlich die organische Staatsidee... sich herausringen wird, desto grössere und vollere Freiheit, selbst auf die praeterita [d.h. Rückwirkung] wird man dem Staat vindizieren".

3 „Ein Staat ist aber ein Organismus, der nicht bloss nach aussen, sondern durch und durch frei ist" (S. 302). „Vor einer folgerichtigen... Deduktion aus dem Prinzip der Willensgemeinschaft kann ein aus dem individuellen Willen geschöpftes Rechtsprinzip nicht mehr bestehen" (S. 303). 
Rodbertus ist als totaler Kollektivist Monist und zeiht Lassalle des versteckten und verschämten Dualismus. ${ }^{1}$ In seinen Augen ist der Rechtsstaat, der vom Kollektiv her Einzelrechte garantiert und durch diese Einzelrechte gebunden ist, nur eine historisch-germanische Erscheinung, die nun mit Hilfe einer zu ermittelnden objektiven Regel, die dem Staat sagt, was Rechtens sei, abzulösen wäre. ${ }^{2}$ Demnach ist Lassalles „System” ein unzureichender Versuch in der richtigen Richtung, denn trotz Lassalles eingehender Darlegung bleibt er dabei, dass bei ihm keine wahre Einheit der Gegensätze von Einzelwillen und Willensgemeinschaft vorliege, sondern dass es sich um eine dialektische Täuschung handle. ${ }^{3}$ Er ist fest davon überzeugt, dass sich - wenn auch in "geologischem" Zeitsabstand - eine objektive, naturwissenschaftliche Gesellschaftslehre formulieren lasse, die der Kraftverschwendung und den Irrwegen der heutigen Gesellschaft ein Ende machen werde.

Bei solcher Erwartung der Endlösung aller Gesellschaftsfragen mittels eines „novum organum" 4 können parlamentarische Abstimmungen, wie selbst Volksabstimmungen nur ein Notbehelf sein, denn auch Einstimmigkeit ist nach seinen Vorstellungen vom sozialen Körper und seinen individuellen Atomen ${ }^{5}$ nur Entscheidung einer „Partei”. ${ }^{6}$ So kann er denn auch im Gegensatz zum Dreiklassenwahlrecht des allgemeine und gleiche Wahlrecht billigen, aber doch niemals Demokrat sein. In seinen Augen gilt die Souveränität objektiver Wissenschaft und nicht die Souveränität subjektiver Volksmeinung.

Lassalle aber ist Demokrat. Auch für ihn ist das heutige Zeitbewusstsein objektiv gegeben, aber daraus zieht er einen ganz anderen Schluss. Er antwortet Rodbertus: "Wie finde ich dies [das objektive Zeitbewusstsein] also? Nun ich denke ganz einfach! Was Sie sich und der Zeit durch Vernunft, Logik, Wissenschaft beweisen können - dass will die Zeit!" ?

1 S. 303.

2 S. 304 .

3 „Individueller Wille und Willensgemeinschaft sind gar nicht solche Gegensätze..., deren jeder den anderen in sich trüge. Der erstere lässt sich durchaus ohne den letzteren denken" (S. 318).

4 S. 305 .

5. 318 .

- S. 304. „Ich erlaube mir den Vorwurf, dass Sie über diese höchste und heiligste - ich möchte sagen heimlichste - Frage der [Rechts] Materie im Grunde nur den Sieg der Partei entscheiden lassen”. Hier wird „Partei” noch im vorparlamentarischen Sinn als etwas dem sittlichen Ganzen entgegenstehendes verstanden.

7. 3 I3. 
Die zu Bewusstsein und Klärung gelangte Wissenschaft hat die Pflicht der Propaganda, deren Ergebnis "richtige” demokratische Meinungsäusserung ist. Grundlegend ist dabei die Voraussetzung, dass dieselbe Erkenntnis "an sich" und abstrakt schon vorher im "Volksgeist" bestanden habe, und nun durch die Verbindung von "Wissenschaft" und „Volksgeist” konkret-wirklich werden kann. Diesen Übergang hat Lassalle in Bezug auf neues Rechtsbewusstsein eingehend dargelegt: Objektive Erkenntnis wird bindende Rechtsnorm durch Verbindung mit dem Volksgeist. Diese Erkenntnis war Grundlage von Lassalles demokratischer Agitation, die der Arbeiteragitation voranging.

Der Zeitwille entfaltet sich im Lauf der Geschichte, dem man nicht vorgreifen darf. ${ }^{1}$ Schon deshalb lehnt Lassalle es ab, nach einer Norm zu suchen, die diesen Zeitinhalt bis ins Unendliche bestimmen will, so wie sie Rodbertus vorschwebte. Dieser braucht nur eine verfeinerte Gesellschaftslehre, um aller Schwierigkeiten Herr zu werden. Lassalle dagegen braucht ausserdem den fortlaufenden historischen Prozess, in dem die Wissenschaft allmählich zum Selbstbewusstsein kommt, und zudem noch auf jeder Stufe das demokratische Element der Massenüberzeugung, die allein schlüssiges Handeln sanktionieren kann. ${ }^{2}$

Dass der zeitbedingte Inhalt des Volksbewusstseins sich öffentlich aufweisen muss, - das ist der demokratische Grundstock in Lassalles Lehre, der bei Rodbertus wegfällt. Jene Massenüberzeugung bewirkt die Einheit von persönlicher Willensfreiheit und Willensgemeinschaft; sie bewirkt, dass das Individuum nur im Rahmen des vom Kollektiv Gesetzten und Gebilligten „will”; sie ermöglicht im Kollektiv die Demokratie. Willensgemeinschaft, metaphysischer Glaube an Volksgeist, „volonté générale” oder Standes- und Klassenbewusstsein, besagt aber wiederum: Pflicht der Propaganda. Damit ist das Hauptmerkmal des zu gründenden Arbeitervereins vorgezeichnet.

Mit diesem Grundbegriff der Einheit von Einzelwillen und Gemeinschaft, den Lassalle von seiner demokratischen Aktion auf den Arbeiterverein übertragen will, setzt sich Rodbertus sowohl logisch

IS. 312. „Die Fragen... welchen Inhalt jedes spätere Zeitbewusstsein haben wird, sind offenbar Fragen, deren inhaltliche Beantwortung durch keine Regel - die wäre ja ein reines Vademecum für die ganze Weltgeschichte! - gegeben werden kann". Rodbertus hat ein solches Vademecum - und entsprechend seiner naturwissenschaftlichen nicht historischen Auffassung mit Recht - erwartet (S. 317 ). Anders Lassalle als Hegelianer.

2 Solche demokratischen, nicht parlamentarischen Massenhandlungen, die neue Rechtserkenntnisse und Rechtsbasen schaffen, liegen der Darstellung der Geschichte der sozialen Entwicklung (Bd. 6, S. 89-155) und dem ersten Teil des „Systems" zugrunde. 
wie historisch auseinander, indem er nachweist, dass dieses Verhältnis kein wirklich zweiseitiges ist, wie es die Dialektik fordere, und dass auch historisch das eine ohne das andere bestanden hat. ${ }^{1}$

Der Dualismus von Einzel- und Gemeinwillen, den Rodbertus monierte, ist bei Lassalle wirklich vorhanden, trotz gegenteiliger Beteuerungen. Das „Offene Antwortschreiben”, das eben in den Tagen der Auseinandersetzung entstand, ist dafür ein klarer Beweis. Der Kollektivwille, der sich im Staate ausdrücken sollte, wird in diesem zur grossen Assoziation der bedrückten Schichten degradiert, und zugleich wird die freiwillige, individuelle Assoziation der Werktätigen proklamiert: In beiden Fällen kann nur vor einem Auseinanderfallen und nicht von Einheit von Einzel- und Gemeinwillen die Rede sein. ${ }^{2}$

Rodbertus hat den letztlich liberalen Ausgangspunkt von Lassalles Denken schon im „System" entdeckt und hatte versucht, ihn zu bekämpfen; nun muss er feststellen, wie dieses individuelle und liberale Element im „Offenen Antwortschreiben" beherrschend wird: Organisierte Arbeitergruppen sollen in Parlamenten um Staatskredite kämpfen, Kooperative aufbauen, um selbst Unternehmer zu werden. ${ }^{3}$

Es ist leicht verständlich, dass Lassalle, dem das „Offene Antwortschreiben" schon vor Augen schwebte, die theoretisch so bedeutsame Diskussion über die letzten Grundlagen des ,Systems der erworbenen Rechte" nicht weiter geführt hat. Schon seine Darlegungen im dritten Brief sind mehr eine Zusammenstellung von Zitaten aus dem „System” als eine wirkliche Exposition. ${ }^{4}$ Selbst Rodbertus' Skizze einer künftigen Vollphilosophie, die die heutigen „Drittelphilosophie” ersetzen sollte, hat ihn nicht zu weiterer Diskussion verführt. ${ }^{5}$ So hat Lassalle es vermieden, die Grundlagen seiner Philosophie in Frage zu stellen, eben weil er sie in der praktischen Agitation beiseite schob. In der Theorie gibt es keine Brücke zwischen Gesetzgebung als Ausdruck eines Kollektivbewusstseins und sozialer Aktion zugunsten einer besonderen, wenn auch noch so breiten Volksschicht. Es kommt auf

1S. 318-319.

2 F. Lassalle, Gesammelte Reden und Schriften, hrsg. und eingel. von Ed. Bernstein, Berlin I919, Bd. III, S. 88. Bis zum „Offenen Antwortschreiben” war der Staat Gesamtwillen, hierarchisch dem Besonderen übergeordnet. Eine neue Idee muss die Gesellschaft sprungweise erobern, wie im „Arbeiterprogramm" die "Idee des Arbeiterstandes”.

3 S. 69 .

4 Nachgel. Briefe, Bd. 6, S. 318-319.

5 S. 320-321. Ende von Brief 4. Die Kritik Rodbertus' klingt an Marx' These r I über Feuerbach an, und hätte den Panlogiker Lassalle anreizen müssen, wäre er geneigt gewesen seine geistigen Grundbegriffe zu überprüfen. 
Wahrung des hierarchischen Stufenverhältnisses zwischen Gemeinund Einzelwillen an.

Schon der Auftakt des Briefwechsels hat eine tiefe Krise der Theorie beim Übergang von demokratischer und sozialdemokratischer Aktion erwiesen, die sich praktisch dahin auswirkt, dass Rodbertus als strenger Staatsmonist der zu gründenden Bewegung fern bleibt.

\section{IV}

Das „Offene Antwortschreiben” gipfelt in der Forderung, dass die Arbeiter sich organisieren sollen, um das demokratische Stimmrecht zu erkämpfen und mit dessen Hilfe Staatskredite für genossenschaftliche Produktion zu erzwingen. Lassalle hat es in einem berühmt gewordenen Brief an Gustav Lewy sehr dramatisch darzustellen gewusst, wie Franz Ziegler vor der sozialen Seite des Programmas zurückgeschreckt sei; ${ }^{1}$ die längst bekannten Briefe Lassalles an Rodbertus aber geben die Kehrseite: Rodbertus schreckt vor der politischen Forderung zurück. Lassalle allein also besteht auf der organischen Einheit der sozialen und politischen Aktion. Nach Veröffentlichung der Briefe Zieglers an Lassalle im 5. Nachlassband und der Briefe von Rodbertus kann nun nachgewiesen werden, dass Lassalle die wahre Sachlage aus agitatorischen Gründen sehr einseitig dargestellt hat.

Für Ziegler bestand sicherlich der Primat des demokratischen Elements, wie für Rodbertus der des sozialen. Beide haben aber mit Lassalle um eine richtigere Einordnung beider Elemente gerungen, ihm die theoretische Unhaltbarkeit seiner spezifischen Interpretation beider Elemente, und insbesondere die Art und Weise von deren Verknüpfung vorgeworfen.

Rodbertus' Name hatte zuviel Gewicht um ein ähnliches Verfahren ihm gegenüber $\mathrm{zu}$ rechtfertigen, wenn das kompliziertere, das er dann wählte, tatsächlich auch zum selben Ergbenis führte. Lassalle wollte für den Übergang von demokratischer zu sozialdemokratischer Aktion eine organische Einheit behaupten, deren Unwirklichkeit Ziegler und Rodbertus durchschaut haben.

Lassalle kam zur Arbeiteragitation erst nach dreijährigem Ringen um die Errichtung einer demokratischen Partei. Noch im „Arbeiterprogramm" hat er den Versuch unternommen, das demokratische Erbe einfach dem Arbeiterstand als dem natürlichen Träger demokratischer Tugenden zu übertragen. Erst im „Offenen Antwort-

1 Bd. 5, S. Iog-ıio. Dasselbe fast wörtlich auch an die Gräfin Hatzfeld, ein deutliches Beispiel von ,Selbstüberzeugung." 
schreiben" wird ein Bruch mit der Vergangenheit vollzogen, in dem versucht wird, den demokratischen Gedanken mit einem Sonderinteresse zu verbinden, die „unendliche" Idee für einen „endlichen” Zweck einzuspannen.

Die demokratische Idee kennt aber nur unerbittlichen Kampf gegen jedes Privileg und jede Absonderung. So musste denn Lassalle als Demokrat den Nachweis erbringen, dass letzten Endes das Sonderinteresse des Arbeiterstandes im allgemeinen Interesse aufgeht, und diesem Ziele sollte die sehr merkwürdige Statistik dienen, nach der 96\% der Nation „Arbeiter" seien, die dadurch den Staat ausmachen und für die deshalb Staatshilfe in Selbsthilfe umschlägt. ${ }^{1}$ Nun sind in dieser Statistik alle Arten gedrückter Lebenshaltung zusammengewürfelt, sodass nicht nur die begriffliche Ableitung fehlt, die ihm doch bis dahin als Beweis wahrer Wissenschaft galt, sondern selbst eine gewöhnliche Klarheit der Begriffe. Daher stehen in Wahrheit bei Lassalle Sozialdemokratie und Demokratie in schroffem Gegensatz wie Gruppeninteresse und Gemeinwohl. Diese Inkongruenz hat Rodbertus klar durchschaut, wenn er von den Weizenbodenassoziierten spricht, die eine gemeinsame Front gegen die Assoziierten schlechtere Böden bilden, um ihren Vorteil zu schützen. ${ }^{2}$ Er spricht vom Kollektivegoismus der schlimmer und zäher sei als der Egoismus von Einzelnen; er malt das Schreckbild eines konstitutionellen Staates im Massstab eines jeden Einzelbetriebes. ${ }^{3}$

Diese Kritik musste Lassalle empfindlich treffen, denn während der drei Jahre demokratischen Kampfes hat er im Namen der einen unteilbaren solidarischen Gesellschaft jeden Partikularismus, Föderalismus und Konstitutionalismus bekämpft; er hat aufgezeigt, wie die liberale anarchische Gesellschaft den Staat zersetzt, ihn partikularen Interessen unterwirft, um ihn schliesslich in der Gesellschaftsanarchie aufzulösen. Nun zeigt Rodbertus, wie gerade dies in erhöhter Potenz der Effekt seines individuellen Assoziationssystems sein muss.

In der Diskussion um die letzten Grundlagen des „Systems” hatte Rodbertus das nur Scheinbare der spontanen Einheit von Einzelwillen und Gemeinwillen bei Lassalle erwiesen; nun zeigt er in der aktuellen Auseinandersetzung mit dem „Offenen Antwortschreiben” die Illusion der Einheit von Gruppeninteresse und Gemeinwohl.

1 Reden und Schriften, Bd. III, S. 80-8I.

2 Nachgel. Briefe, Bd. 6, S. 364 , Nr. 28. „Kollektivpersonen, wie sie ein zäheres Leben haben als Individuen, haben auch einen zäheren und härteren Eigennutz".

3 S. 316. "Jeder Betrieb... ein kleiner konstitutioneller Gewerbestaat..., in dem jeder Arbeiter von Rechtswegen mitzureden hätte". 
In der Kontinuität des Meinungsunterschiedes ist methodisch und prinzipiell der Trennungsstrich zwischen Rodbertus und Lassalle gezogen; die Frage der Assoziationen ist nur ein Sonderfall. Falsche Staats- und Gesellschaftsauffassung, die einer Klärung ausweicht, ist Ursache genug für Rodbertus' Fernbleiben. So ist auch die Stimmrechtsfrage Symptom, nicht Ursache.

Lassalle war fest entschlossen, sich durch nichts von der taktischen Verbindung von Wahlrecht und sozialem Interesse abbringen zu lassen. Die Gründe liegen zunächtst nicht in den sozialen Notwendigkeiten der Arbeiterbewegung, sondern in der politischen Situation des Verfassungskonfliktes.

Lassalle glaubte zur Zeit seiner letzten Verfassungsaufsätze im Winter des Jahres $1^{86} 3$ und bis zu seiner ersten Unterredung mit Bismarck im Sommer an die Möglichkeit eines revolutionären Durchbruchs. Diesem Glauben hat er Theorie und Organisationschancen geopfert. Es blieb ihm dabei nur die Möglichkeit des taktischen Lavierens. In der zweiten Briefgruppe kommt dies klar zum Ausdruck.

Auf drei Wegen hat Lassalle versucht, Rodbertus' Gegnerschaft zu seinem sozialen Programm zu überwinden, oder mehr noch: zu überlisten.

Der erste Weg war, sich und Rodbertus einzureden, dass eigentlich gar keine wirkliche Gegnerschaft vorliege. Dieser Weg ist für den Menschen Lassalle besonders bezeichnend. ${ }^{1}$ Solche Versuche haben Rodbertus $z u$ immer schärferer Stellungnahme gezwungen, so dass gegen Ende der Korrespondenz sich Lassalles soziales Mittel als das erweist, was es an sich schon zu Beginn war: Das eigentlich Trennende. ${ }^{2}$ Wirklich kann es keinen grösseren Unterschied in der Auffassung des Sozialismus geben, als die prinzipielle Bejahung der Lohnarbeit durch Rodbertus, wenn nur gerechter Lohn gezahlt wird, und die ebenso prinzipielle Ablehnung der Lohnarbeit durch Lassalle, der die Arbeiter zu eigenen Unternehmern machen will um einen neuen Mittelstand zu schaffen. ${ }^{3}$ Eine merkwürdige Selbsttäuschung hat Lassalle dazu verleitet, den Schlusssatz von Rodbertus' Aufruf an die Leipziger, in dem seine Gegnerschaft scharf hervortrat, zu streichen. Die

${ }^{1}$ S. 335 . Die geharnischte Antwort in Nr. 17, S. 342.

${ }^{2}$ S. 375, Nr. 35.

${ }^{3}$ Reden und Schriften, Bd. III, S. 72. 
Erörterung dieser Streichung ist wohl die peinlichste Stelle der ganzen Korrespondenz. ${ }^{1}$

Lassalles zweiter Weg besteht in einer scheinbaren Bereitschaft zum Verzicht auf die Assoziationsidee. Lassalle stellt es so dar, als sei er einer Augenblickseingebung gefolgt und dementsprechend bereit, jedes andere Mittel vorurteilslos zu prüfen, wenn es nur konkret demokratisches Stimmrecht und soziales Lebensinteresse der Arbeiter verbinde, denn eben auf diese Verknüpfung kommt es ihm an. Diese scheinbare Bereitschaft hat Lassalle recht unorganisch in die grosse Frankfurter Rede als deutliche Verbeugung vor Rodbertus eingeschoben. ${ }^{2}$

Lassalle brauchte nicht auf Marx' Inauguraladresse zu warten, um den sozialen Wert der gesetzlichen Verkürzung der Arbeitszeit zu erkennen; er kannte das Kommunistische Manifest mit seinem Übergangsprogramm; er wusste, dass ein ro-Stunden Bill und ein Verbot der Frauenarbeit für das Gesamtproletariat mehr bedeuten als Dutzende von Kooperativen; er hatte im „System” selber den Weg revolutionärer Gesetzgebung vorgezeichnet und dessen Grundlagen mit Rodbertus erörtert; er wusste, dass dieser gezetzliche Regelung des Normalarbeitstages und des Normalarbeitslohnes verlangte.

Wenn er trotzdem auf der Assoziationsidee beharrte, so bestimmten ihn zwei Umstände: Er war gar nicht frei, wie er vorgab, und das wahre Verhältnis von demokratischem Mittel und sozialem Ziel war ganz anders, als er es Rodbertus darzustellen versuchte.

Lassalle hat Anfang $186_{3}$ aus demokratischen Motiven in eine gärende, eigenwillige und sich in schnellem Tempo klärende Arbeiterbewegung eingegriffen. Verschiedene Monographien lokaler Arbeiterbewegungen erzählen übereinstimmend, wie genau um dieselbe Zeit eine Arbeiterkongressbewegung das System geschlossener Vereine abzulösen begann, wie der Unterschied zwischen Lohnarbeitern und Handwerkern klarer zutage trat, wie aber alle Teile der Bewegung in Assoziationen verschiedener Art das Mittel des Heils sahen: Das waren die grossen Tage eines Schulze-Delitzsch. Man erhoffte einen Ausweg aus dem Lohnsystem: Der Handwerker wollte dem Schicksal des Lohnarbeiters entgehen; der Proletarier hoffte auf Rückkehr in den Mittelstand. ${ }^{3}$

1 "Und ich wiederhole, dass ich mir auch von den Produktivassoziationen nicht im geringsten einen Beitrag zu dem verspreche, was man die Lösung der sozialen Frage nennt." S. 324. "Sie sollen Indemnität haben" - S. 326.

2 Reden und Schriften III. S. 243. „Nur um das Ptinzip handelt es sich heute”.

3 Besonders: Laufenberg, Geschichte der Arbeiterbewegung in Hamburg, Altona und Umgegend, Hamburg I9I I, S. 203-17. Auch die Einleitung von Bernstein, Reden und Schriften Bd. III, S. I 2-20; 23-25; 47-49. 
Nach drei Jahren demokratischer Agitation, die aber zu keiner demokratischen Sammlung führte, hat Lassalle versucht, diese soziale Bewegung für seine demokratisch-revolutionären Ziele einzuspannen. Dies ist ihm auch zum Teil gelungen, nur dass die revolutionären Ziele selbst durch die schleppende Taktik der Fortschrittler und durch das Scheitern der Bismarckgespräche hinfällig wurden. Um die Bewegung seinen Zielen dienstbar zu machen, musste er sie überrumpeln, aus ihrer inneren Entwicklung herausreissen und ihr neue Kampfparolen geben. Dank seiner intellektuellen Überlegenheit fiel es ihm leicht, überzeugend darzulegen, dass alle die Grundrechte und Freiheiten, die die Arbeiter mit Hilfe der Liberalen zu erreichen hofften, entweder selbstverständlich, unwichtig und vielleicht sogar fragwürdig seien; dass aber der Traum des ,vollen Arbeitsertrages" sich nur gegen die Fortschrittler und mit Hilfe des durch das demokratische Wahlrecht regenerierten Staates erfüllen lasse. Nachdem Lassalle die Nichtigkeit des Kongressprogramms nachgewiesen hatte, fiel es ihm nicht schwerer als Marx in ähnlichen Umständen, sein eigenes Programm einzuschieben. Aber auch jetzt musste das Assoziationsprogramm den Mittelpunkt bilden - und daran hat sich fast bis Gotha nichts geändert, sowohl bei Lassalleanern wie bei den "Internationalen". ${ }^{1}$

So ist denn auch die Frankfurter Rede ganz von dem Assoziationsgedanken getragen, und Lassalles Geste hat Rodbertus mit Recht nicht überzeugt. Im „Bastiat-Schulze” hat dann Lassalle noch einmal den Assoziationsgedanken in messianische Höhen erhoben.

Die Schwäche eines Schulze-Delitzsch war die Stärke Lassalles: Es war leicht zu beweisen, dass durch Sparen die Assoziation im Grossen nicht zu bewerkstelligen war; aber es war schwer, die entwickelteren Arbeiter zu bewegen, sich mit dem verhassten Obrigkeitsstaat einzulassen. Die von Lassalle geforderte Verbindung von demokratischem Stimmrecht mit der Assoziationsidee war nicht überzeugend, und Lassalles „Stimmrechtsarmee” blieb klein, aber nicht aus Mangel an sozialem und politischem Selbstbewusstsein, sondern um ihrer selbst wegen. Es war die allgemeine Überzeugung, dass das demokratische Stimmrecht in der gegebenen politischen Lage nicht an der Tagesordnung sei, aber leicht $\mathrm{zu}$ antidemokratischen und antiliberalen Manövern ausgenützt werden könne. Deswegen sollte der Assoziationsgedanke, der einer der stärksten Impulse im sozialen Leben geworden war, eingespannt werden, um der politischen Bewegung

1 Bracke war der erste der den Bann der Assoziationsidee in der sozialistischen Bewegung brach. „Der Lassallesche Vorschlag”, Braunschweig 1873 . Gegen Ende des Jahrhunderts hatte die Idee der Verstaatlichung den Assoziationsgedanken ganz verdrängt. 
eine Wendung zu geben die von Vielen gefürchtet wurde. Aber es war ja nicht einmal das Stimmrecht an sich. Dies war vielmehr nur Signal zur Revolution, denn nach seiner Theorie kann die Bourgeoisie auf das Wahlrechtsprivileg nicht verzichten. So wurde der Assoziationsgedanke Mittel zum Mittel, schien aber als solches unentbehrlich.

Demokratisches Wahlrecht und soziale Gesetzgebung waren logisch und historisch besser verbunden als Wahlrecht und Assoziation. Es schien aber Lassalle, als biete die Gesetzgebung nichts, was nicht schon in der demokratischen Agitation benutzt worden wäre, und wie es schien - vergeblich.

Nachdem die Aussichten auf politischen Durchbruch schwanden, konnte die unnatürliche Verbindung von Assoziationsgedanken und Stimmrecht gelockert werden, und der Assoziationsgedanke selbst mehr als Selbstzweck zu seinem Rechte kommen. Aber die Verkuppelung zu Beginn der Agitation hatte ihren unheilvollen Einfluss schon ausgeübt: der beste Teil der Arbeiterbewegung blieb der Lassalleschen Organisation fern und auch Rodbertus blieb weiter der "Theoretiker von Jagetzow".

Es bestand noch eine dritte Möglichkeit der Annäherung an Rodbertus, die praktisch an seiner Haltung nicht viel geändert hätte, aber, wenn ehrlich durchgeführt, der sozialen Aktion eine theoretisch haltbarere Basis ergeben hätte: Lassalle konnte den Assoziationsgedanken selbst reformieren und Rodbertus' gerechtfertigten Einwendungen nachkommen.

Im „Offenen Antwortschreiben” entsprach die Auffassung der Assoziationen den Wünschen des Handwerkers, der Lohnarbeiter geworden war und nun die Frucht ,,seiner” Arbeit in fremden Händen sieht und selber am Rand des Existenzminimums lebt. Er verlangt seinen (individuellen) vollen Arbeitsertrag. Zu diesem Zweck muss er die Produktionsmittel, mit denen er tagtäglich umgeht, in seine eigenen Hände bekommen. Es war Lassalles Verdienst gewesen, eben diesem Lohnarbeiter mit Handwerkergesinnung, die Aussichtslosigkeit des Kleinhandwerks bewiesen zu haben, sodass er jetzt einsah, dass die Mittel der modernen Grossproduktion nur in Gemeinschaft und Einverständnis mit seinen Kollegen verwaltet werden können und mit öffentlicher Hilfe: Jede Kooperative organisiert sich spontan und geht dann den Staat um Kredit an; nur das gute Beispiel und der grosse Vorteil wirken. ${ }^{1}$

${ }^{1}$ Reden und Schriften, Bd. III, S. 69. 
Dagegen hatte Rodbertus eingewandt, dass industrielle Produktivassoziationen nicht genügen, dass Landwirtschaft und Transport folgen müssen, dass einzelne, auf sich selbst gestellte Kooperativen das Problem kapitalistischer Krisen nicht lösen können, dass das Regime parlämentarischer Leitung die Kooperativen zu schwerfälligreibungsvollen und hässlichen Gebilden machen müsste. ${ }^{1}$ Lassalle hat diesen Einwendungen gegenüber sofort nachgegeben und erklärt, dass nichts der Ausdehnung der Kooperativen über das Gebiet der industriellen Produktion hinaus im Wege stände, dass das Krisenproblem sich lösen liesse, sobald die Kooperativen sich branchenweise assoziieren und dadurch Marktforschung ermöglichen, und jedem Leitungsparlamentarismus ein Ende gesetzt werden kann mittels authorisierter Geranten, deren Handhabung der Geschäfte Autorität und Disziplin verbürgen. ${ }^{2}$

Mit dieser neuen Auffassung war es Lassalle völlig Ernst, wie seine späteren Äusserungen über das Assoziationswesen immer diese neue Interpretation zulassen, und wie besonders ein Entwurf für die Statuten einer Buchdruckerassoziation beweist, der im 6. Nachlassband abgedruckt ist. ${ }^{3}$

Die Brücke zu Rodbertus hat Lassalle damit aber nicht geschlagen, da dieser als wahrer Staatssozialist gleiche Abhängigkeit Aller vom Staat erstrebt, Alle Lohnempfänger vom Staat werden sollen und jedes Mass wirtschaftlicher Selbständigkeit von Übel ist. Zugleich hat sich Lassalle aber mit der Reform des Assoziationsgedankens von den Idealen der Arbeiter entfernt, die diese nur als wahrhaft individuelle, spontane und demokratische Schöpfung erstrebten.

Wie in der Diskussion um die Grundlagen des "Systems" schon theoretisch, so tritt hier nun das Auseinanderfallen von Einzel- und Gemeinwillen praktisch in Erscheinung: Die Synthese von Freiheit und Autorität erweist sich als rein vorgestellt und erfunden.

\section{VI}

Die Arbeiter, an die das „Offene Antwortschreiben” gerichtet war, verlangten freiwillige Assoziation und freie demokratische Führung der Geschäfte; sie wollten Herren des gemeinsamen Einkommens sein. Lassalle hat ihnen auch nachdem er Rodbertus die wichtigen Zugeständnisse gemacht hat, nie offen gesagt, dass man für den Besitz schlechteren Landes oder für entferntere Märkte entschädigt

${ }^{1}$ Nachgel. Briefe, Bd. 6, S. 316.

2 S. 324-325.

3. $396-398$. 
werden müsse, oder dass sich also im Einzelfall der „volle Arbeitsertrag" nicht realisieren lasse. Er hat eine branchenweise Regulierung in seine Darstellung eingeschoben, aber nie erklärt, dass diese Regulierung Pflicht zu sein hat und planmässig erfolgen muss, um zum Ziel zu führen; dass also Zwang gar nicht zu vermeiden ist. Lassalle hat den Arbeitern nie erklärt, dass die „Diktatur der Einsicht”, die im "Allgemeinen Arbeiterverein" herrschte, nicht nur praktische Notwendigkeit ist, sondern einem Gesellschaftsideal entspringt und auch für die Leitung der Kooperativen massgebend sein soll, dass also der Gerant genau wie der Präsident des Vereins einmal im Jahr gewählt wird, dann aber uneingeschränkt handelt und sich nur vor seiner Mandatserneuerung zu verantworten hat.

Die endgültige Verfassung des Assoziationswesens ist also weit entfernt von den ausgesprochenen Idealen der deutschen Arbeiter, deren Willen auf diese Weise denaturiert wird, ist aber doch kooperativ in dem Sinne, dass prinzipiell dem Arbeiter der volle persönliche Arbeitsertrag zusteht, sei er auch praktisch um eine ganze Anzahl von Abzügen geschmälert, an die der Arbeiter nie gedacht hatte. Rein theoretisch gesehen, bestätigt Lassalles Wertlehre im „BastiatSchulze" dieses Anrecht einer jeden Arbeitergruppe auf den vollen Wert ihrer konkreten Produktion. ${ }^{1}$ In der Schau von Rodbertus aber gehört jedes Produkt gesellschaftlicher Arbeit dem Staat, der dann jedem das Seine gibt. Er braucht keinen Glauben an einen besonderen Gemeinwillen, um sein System in Gang zu setzen. Die gesellschaftlichen Verhältnisse wie sie heute in Fabriken und Gutshöfen bestehen, sind in seinen Augen normal, sobald jeder das bekommt, was ihm zusteht. Das festzustellen ist Aufgabe der Nationalökonomie, die damit Staatswissenschaft wird.

Lassalle weiss, dass der zufällige, willkürliche Einzelwille in Staat, Verein und Assoziation zu Gunsten höherer Ziele umgebogen werden muss; aber er glaubt, dass damit ein metaphysischer "wahrer" Einzelwille gerade durchgesetzt wird. Dass dieser Glaube tief bei ihm verankert ist, beweist er durch sein Festhalten an dem Prinzip demokratischer Wahlen, auch nachdem es ihm keinen politischen Vorteil mehr bietet. Aber diese Wahl muss als feierlicher Bestätigungsakt des Gemeinwillens verstanden werden, als Ausdruck des Volksgeistes, der in jedem unverfälschten Bürger haust; nie und nimmer soll sie Auftakt zu parlamentarischem Kuhhandel sein. Weil "wahrer" Einzelwillen und "wahrer" Gemeinwillen zusammenfallen, kann Lassalle systematisch vom Einzelwillen ausgehen, und selbst den

1 Tatiana Grigorivici: Die Wertlehre bei Marx und Lassalle, in: Marxstudien Band III, Wien I9ro. 
Widerspruch zwischen seinem „System” und dem „Offenen Antwortschreiben" nicht klar bemerken.

Rodbertus stellt bei Lassalle den Ausgangspunkt vom liberalen Denken her fest; in der Erörterung des Assoziationsgedankens beweist er es in dessen Verhältnis zu den Begriffen von Lohn und Gewinn. ${ }^{1}$ Lohn ist für Rodbertus einfach Bestätigung von Leistung ${ }^{2}$ im Sinne eines „suum cuique”, für Lassalle aber Ausfluss der menschlichen Entfremdung. Dieser Gegensatz lässt sich nicht überbrücken. Für Rodbertus reduziert sich die ganze soziale Frage auf richtige Entlohnung individueller Leistung durch die Staatsgesellschaft.

Zwischen der Körperschaft des Ganzen und dem tätigen Einzelnen hat sich ein Zwischenglied eingeschoben, die bürgerliche Gesellschaft, die sich ein verfälschtes Wertsystem geschaffen hat, in dem Begriffe wie Gewinn, Kapitalprämie, Entbehrungslohn und Risikoprämie spuken. Dieses verfälschte Wertsystem muss begrifflich durchschaut und dann allmählich abgebaut werden. Dann bleiben Einzelzelle und Staatsorganismus: Die gesamte Gesellschaftslehre seit Hegel ist wie weggeblasen. Hier liegt wirklicher totaler Staatssozialismus vor.

Für Lassalle ist die soziale Frage ein Problem der Emanzipation. Die Entfremdung des Menschen in der bürgerlichen Gesellschaft muss überwunden werden; das Kapital muss befreit, der Gewinn erobert werden; Dienst und Lohn müssen verschwinden. In aller Naivität umschreibt Lassalle Rodbertus' Ziel als „dem Arbeiter in Fabrikation und Handel eine Gewinnquota sichern" und setzt dies gleich mit dem ,natürlichen Arbeitslohn", der ,das ganze Produkt der Arbeit" ist. ${ }^{3}$ Erst auf Grund von Rodbertus' Einwendungen bestätigt Lassalle, dass auch seiner Ansicht nach der Arbeiter zunächst laufend Lohn erhält, da der Gewinn sich erst am Jahresende feststellen lässt, aber Lohn und Gewinn bilden zusammen den vollen Arbeitsertrag, den er nie und nimmer „Lohn” nennen möchte. „Ich will also gerade den ganzen Arbeitsertrag den Arbeitern zuwenden, indem ich auch den Geschäftsgewinn ganz unter sie verteile." " Damit ist aber nicht nur der Proletarier zu eigenem Unternehmer geworden, sondern auch ein menschliches Verlangen erfüllt und die Bewältigung des Problems der menschlichen Entfremdung zu mindest in Angriff

1 Nachgel. Briefe, Bd. 6, S. 342. „Sie schleppen des Gefängnisses Schmach...” solange Lassalle den Begriff von Gewinn beibehält.

2 S. 35 2. „Denn die Natur selbst hat den Arbeitersertrag als Lohn der Arbeit hingestellt”.

3 S. 334 .

4 S. 348 . 
genommen. ${ }^{1}$ Lassalle fordert, dass der Staat kulturfördernd wirke, nicht kulturschaffend. Er soll der Gesellschaftsinitiative hilfreich entgegenkommen; Kooperativen und deren Assoziation sollen aber autonom bleiben. Wenn der Arbeiter Herr seiner Produktionsmittel geworden ist, so verliert das Geld seine abstrakte, gegenständliche Qualität; es hört auf, den Produzenten von den Gütern zu trennen. ${ }^{2}$ So wird innerhalb der autonomen Gesellschaftssphäre die Aufhebung der Entfremdung angebahnt: Echter Staatssozialismus ist das nicht!

Angesichts dieser Vorstellungen rüttelt nun Rodbertus das nationalökonomische Gewissen Lassalles auf. Lassalle muss zugeben, dass solange dem Arbeiter Boden, Kapital und Arbeitsprodukt gehören, von einer Lösung der sozialen Frage nicht die Rede sein kann. ${ }^{3}$ Deshalb ist er gezwungen, eine Zweistufentheorie zu erfinden, derzufolge zunächst mit Hilfe von Kooperativen das soziale Elend gelindert wird, dann aber in zweiter Phase, die soziale Frage wirklich gelöst wird: Den Kooperativen werden Grund und Boden, Produktionsmittel und Erzeugnis entzogen, damit der Staat ausgleichende Gerechtigkeit üben kann. Der Anteil des Arbeiters wird dann als Anteil an der gesellschaftlichen Gesamtproduktion festgestellt. ${ }^{4}$ Im Endstadium sind wir dann bei Rodbertus' Normalarbeitstag und seinem Normallohn angelangt. Aus Mangel an nationalökonomischen Argumenten muss sich Lassalle Rodbertus beugen.

Im Endstadium löst Rodbertus' Staatsprinzip das Lassallesche Gesellschaftsprinzip ab; der freie assoziierte Produzent wird Staatslohnempfänger. Eine Errungenschaft wird der anderen geopfert. Ein solches antagonistisches Verhältnis nennt aber Lassalle „Lösung” und „Anbahnung der Lösung”. Die Krise der Theorie drückt sich praktisch aus: Der ausgesprochene Wille der sich assoziierenden Arbeiter wird in sein Gegenteil verkehrt, Lassalles eigener Theorie über die Rückwirkung zu Schanden.

Lassalle hat die staatssozialistischen Gedanken, die Rodbertus' Logik ihm aufzwang, nicht ausgearbeitet. Im „Bastiat-Schulze”, dem „vor-

1 Mehrere Manuskriptbriefe des jungen Lassalle sind dem Problem der Entfremdung gewidmet. Sieh: Bd. I, Briefe Nr. 21, 22, 23, 55 .

2 Darüber im „Bastiat-Schulze”, Reden und Schriften, Bd. V, S. 288, 296, 299, 305, 315. Im abstrakt-demokratischen Denken geht bei Lassalle zwar die Gesellschaftssphäre im Staat als dem Allgemeinen auf, aber das ist nicht mehr der empirische Staat, der für Rodbertus massgebend ist.

3 Nachgel. Briefe, Bd. 6, S. 355.

4. 362 . 
läufig endgültigen Kodex der Bewegung" 1 spielen sie keine Rolle. Dort feiert der Gedanke der freien spontanen Assoziation seine Apotheose. In Lassalles letzter literarischen Produktion, in der überaus wichtigen Antikritik zum „Bastiat-Schulze”, wird der Assoziationsgedanke zusammenfassend zur Theorie eines Mittelstandes der Zukunft erhoben. ${ }^{2}$

Wie in allen anderen Entscheidungen, sind für Lassalle die Jugendideale massgebend geblieben: Der Jugendtraum, dass Kommunismus Besitz für Jeden bedeute, hat über die Logik eines Rodbertus den Sieg davongetragen. Seit seiner Auseinandersetzung mit Rodbertus war Lassalle darauf bedacht, von seinen Assoziationen immer nur als "Anbahnung" und „leichtestem Ubergangsmittel" zu sprechen. Unter „Lösung der sozialen Frage” müssen wir uns, der Logik der "Antikritik" entsprechend, gesetzgeberische Massnahmen im Sinne des "Systems" vorstellen, denen zufolge auf Grund eines neuen Rechtsbewusstseins auch die bestebenden Betriebe in Kooperativen verwandelt werden, um den neuen Mittelstand zu schaffen. Im Kooperativsystem ist also im strengen Sinn Lassallescher begrifflicher Ableitung gar keine „Anbahnung” gegeben. Die Krise der Theorie liegt deutlich zu Tage.

\section{VII}

Als Lassalle den Assoziationsgedanken aufnahm, sollte er „Magenwärme" für das demokratische Ideal liefern. ${ }^{3}$ Deshalb hat Rodbertus mit Recht von Lassalles Stimmrechtsarmee gesprochen. Er hatte verstanden, dass Sinn des erkämpften Stimmrechts nicht sein konnte, in deutschen Teilparlamenten, Kredite für Assoziationen zu votieren. Darum hat er, ohne dass Lassalle dazu direkten Anlass gegeben hätte, Probleme deutscher Einigung, der preussischen Krise und des drohenden Cäsarismus erörtert. Er hat eben den revolutionären Sinn von Lassalles Aktion ebenso gut verstanden wie alle Freunde und

\footnotetext{
1 Zirkular an sämtliche Bevollmächtigten vom is.II.64, zitiert bei Hermann Oncken, Lassalle. Eine politische Biographie. 4. Aufl., Stuttgart und Berlin 1923, S. 425. Abgedruckt: Neue Zeit, Bd. 32, II.

2 Reden und Schriften, Bd. V, S. 380-38r. „Die von mir geforderte Verbindung von Kapital und Atbeit... einen Mittelstand schaff, welcher das ganze Volk selbst umfasst".

${ }^{3}$ Reden und Schriften, Bd. III, S. 91 und 285. „Das grösste Interesse der politischen Freiheit... ein soziales Interesse hinter sie zu werfen".
} 
Feinde; er wusste, dass es um Bürgerkrieg ging. Nur unter diesem Gesichtspunkt sind seine Äusserungen richtig zu würdigen. ${ }^{1}$

Rodbertus schreckte weder vor Eingriffen in die bestehenden Vermögensverhältnisse noch vor einer politischen Revolution zurück, aber er war zu der klaren Erkenntnis gekommen, dass die Zeit für erfolgreiche Revolutionen vorbei sei, dass gescheiterte Revolution aber Cäsarismus bedeute. ${ }^{2}$ Er glaubte an den Primat sozialer Veränderung und sah als nächstes politisches Ziel nicht die Stimmrechtsfrage, sondern die Notwendigkeit, den Fortschritt politisch zu schwächen, um das Manchestertum wirtschaftlich überwinden zu können, um dem Staat die nötige Stosskraft gegenüber den staatszersetzenden Tendenzen des Liberalismus zu verleihen. Dadurch bekommt auch Lassalles politischer Kampf gegen die Fortschrittler Bedeutung, wenn er nur nicht zu einseitig auf die Stimmrechtsfrage zugeschnitten ist. Von totaler Ablehnung der Politik kann keinesfalls die Rede sein.

Rodbertus' Einschätzung der modernen sozialen und politischen Kräfte ist viel realistischer als Lassalles, dessen Revolutionsbild noch ganz ,jakobinisch" wirkt. Er hat verstanden, dass die Bourgeoisie sich einem Cäsar unterwerfen wird, wenn man ihre soziale Furcht zu stark erregt. Deshalb deuten die Zeichen der Zeit auf soziale Diktatur, der nur mutig getroffene soziale Massnahmen vorbeugen können. Aus diesem Grunde ist soziale Aufklärung von grösster politischer Wichtigkeit, und Lassalles Aktion auch vom politischen Standpunkt aus anfechtbar. Wenn die Frankfurter Rede als gediegener anzusehen ist als das "Offene Antwortschreiben" mit seiner betonten Blickverengung, so kämpft Lassalles Stimmrechtsarmee in der Eigenschaft als Bürger gegen liberale Gesellschaftsanarchie, gegen Steuer- und Wahlrechtsprivileg. Nicht der Kapitalist ist Objekt von Klassenhass, sondern der Bourgeois Objekt des Nationalhasses der tugendhaften Bürger. Da aber nach Rodbertus' fester Úberzeugung die Bourgeoisie sich sogleich unter die Fittiche von Bismarck flüchten wird, so hat Lassalle den Kampf verloren, noch bevor er ihn begonnen hat. Bismarck steht so im Dienst von „Monsieur Weltgeist” um eine neue soziale Ordnung einzuleiten. ${ }^{3}$ Es ist also unpolitisch, die soziale

1 Langes Artikel in der "Südd. Zeitung”, von Lassalle in seiner "Ansprache” benutzt (Reden und Schriften, Bd. IV).

2 Nachgel. Briefe, Bd. 6, S. 351. „Die revolutionären Kräfte sind heute schwächer und zersplitterter als vor to Jahren und paralysieren sich deshalb gegenseitig. Und dies ist noch immer die Spalte gewesen, durch welche der Cäsarismus auf die Welt gekommen.”

3. 344 ; S. 35 I. 
Forderung einseitig mit dem Stimmrecht zu verbinden, selbst wenn die soziale Forderung richtig gestellt wäre.

Rodbertus wehrt sich betont gegen den Vorwurf, eine Art „Zentralverein für das Wohl der arbeitenden Klassen” zu erstreben. Er will konkrete soziale Forderungen mit Nachdruck vertreten, also eine soziale politische Partei. ${ }^{1}$ Selbst zu einer losen Verbindung von Wahlrecht und sozialer Frage ist er bereit, wenn auch zu keinem Abhängigkeitsverhältnis. ${ }^{2}$ Was ihn fernhält, ist nach der falschen sozialen Zielstellung, die Unterordnung der sozialen Frage unter ein formalpolitisches Ziel, das nur der Aufreizung, nicht aber dem Durchbruch nützen kann.

Den ernsten Argumenten von Rodbertus kann Lassalle nur seinen „alten Glauben" 3 entgegensetzen. Sein Glaube, dass die Revolution, allen selbstgestellten Prognosen zum Trotz, doch durchbrechen muss, ist unverwüstlich. Er hat weder die Liberalen noch Bismarck so realistisch verstanden wie es Rodbertus tat. ${ }^{4}$

$\mathrm{Da}$ bei einem Staatsmonisten wie Rodbertus das politische Ziel der sozialen Aufklärung selbstverständlich ist, so muss man seine Forderung der Wahrung der Reinheit der sozialen Agitation richtig verstehen. Er warnt in Wahrheit vor politischer Aufreizung und Verkuppelung der sozialen Frage mit taktischen Tageslosungen, die einer wissenschaftlichen Klärung der sozialen Frage entgegenstehen. Nicht mehr als Offenhalten der Diskussion hat er am Vorabend der Frankfurter Entscheidung von Lassalle vergeblich verlangt. ${ }^{5}$ In der Wahlrechtsfrage als Einzelproblem war Rodbertus zu Entgegenkommen bereit, und die Schläge, die Lassalle den Fortschrittlern erteilte, haben ihm ein offensichtliches Vergnügen bereitet. Dass aber Lassalle den Cäsarismus mächtig fördere, stellte er I864 als Tatsache fest.

Rodbertus' Haltung zur Zeit der Vorbereitung des „Bastiat-Schulze” ist besonders aufschlussreich. Lassalle will nach dem Scheitern seiner Bismarckgespräche immer mehr betonen, dass er eine wahre Synthese des Politischen und Sozialen anstrebe bei Primat des Sozialen. ${ }^{6}$ Der

1 S. 337 .

2 S. 323 .

3 S. 358. „In einem Jahre werden wir uns zählen". Dieser Ausdruck ist sehr bezeichnend.

4 Der Ausbruch des Dänischen Krieges hat sein Selbstvertrauen - wenigstens für den Augenblick - erschüttert. Gustav Mayer, Bismarck und Lassalle, Berlin 1928, S. 87 .

In der ersten Panikstimmung scheint er exkannt zu haben, dass das Ausspielen von Liberalen und Reaktion vielleicht doch eine lllusion sein könnte.

s Nachgel. Briefe, Bd. 6, Brief Nr. 17, S. 343.

- S. $377 . \mathrm{Nr} .38$. 
„Bastiat-Schulze” soll der Beweis sein. Rodbertus ist sehr aufmerksam, denn er kann es nicht leicht überwinden, dass die einzige Gelegenheit zu einer „grossen Lehrstunde für das deutsche Volk" vorüber gehen sollte, ohne dass er sein Teil beigetragen hätte. Deshalb ist er weit davon entfernt, die vielen Schwächen des Arbeitervereins als Vorwand für seinen Rückzug zu benützen. Noch im Januar I 864 verspricht er einen „dritten Anlauf”, „falls Lassalles Agitation doch noch wirkliches nationalökonomisches Interesse erwecken sollte". 1

Der „Bastiat-Schulze” ist aber, abgesehen von seinem agitatorischen Wert in der Polemik gegen die Manchesterschule, sehr enttäuschend. In ihm verbindet sich falsche soziale Zielsetzung, ausgedrückt in einer messianischen Erhebung der Assoziationsidee in fast ihrer ursprünglichen Form, mit schädlicher politischer Aufreizung, ausgedrückt in der Drohung mit dem „dumpfen Massentritt der Arbeiterbataillone”, die aber nicht Revolution, sondern Cäsarismus heraufbeschwören. Trotz der Freude über den „Bastiat-Schulze” als Kampfschrift sind für Rodbertus die theoretischen Mängel entscheidend. In seiner Antwort auf das Buch reagiert Rodbertus schon ironisch, indem er in vorsichtiger Andeutung, Proudhon zu Lassalles Vorbild macht. ${ }^{2}$ Im selben Sinne hat sich Rodbertus nach Lassalles Tod, aber dann viel krasser und zum Teil recht übertrieben, ausgedrückt. ${ }^{3}$

\section{VIII}

Der Winter des Jahres ${ }_{1} 86_{3}$ ist vom Verfassungskonflikt beherrscht; der Winter des Jahres 1864 von der Dänischen Krise, in der die innerpolitischen Kräfte praktisch ausgeschaltet waren. Lassalles Agitationslosungen bekamen dadurch einen neuen Sinn, sie waren nicht mehr Auftakt zu revolutionärem Durchbruch, sondern Hebel für Wachstum und Gedeihen der Organisation. Im Zeichen dieses Organisationsprinzips hätte er die Momente von Assoziation und Wahlrecht, wie auch deren Verkuppelung überprüfen und sie den Forderungen von Rodbertus, denen er im Briefwechsel wenigstens teilweise nachgekommen war, anpassen können. Er hat in Rodbertus Hoffnungen erweckt, die sein „endgültiger Kodex” aber zerstört hat.

Solange die Agitation im Zeichen des demokratischen Durchbruchs stand, musste Rodbertus fernbleiben, weil die wahren Ziele der Agitation zu einer Denaturierung des ausgesprochenen Willens

1 S. 376. Nr. 36 .

S. 379 . Nr. 4 I.

3 Briefe und social-politische Aufsätze von Dr. Rodbertus-Jagetzow, herausgegeben von Dr. R. Meyer. Berlin o.J., S. 226. 
der Arbeiter verpflichteten, der Rodbertus nie zugestimmt hätte. So wollte Lassalle nur den Namen von Rodbertus und dessen Autorität einfangen und agitatorisch ausnützen. An dieser Tendenz hat aber auch die Periode der relativen Herrschaft des Organisationsprinzips nichts geändert: Alle Wissenschaft musste durch Lassalle vertreten werden, trotzdem Rodbertus und er ,siamesische Zwillinge" waren. ${ }^{1}$ Der Grund war der, dass der Teil der deutschen Arbeiter, den der „Arbeiterverein" erfasst hatte, zu rückständig war, um ohne die Scheuklappen des „Offenen Antwortschreibens” ruhig seinen Weg zu gehen. In der postulierten Einheit von „Wissenschaft und Arbeiter” musste alle "Wissenschaft" seine spekulative Wissenschaft sein, die aber selber, was die Nationalökonomie betraf, noch ein Postulat der Zukunft war, wie Ziegler klar dargelegt hatte.

Die Herrschaft des Organisationsprinzips war nie vollständig: Lassalle war immer zu neuer Revolutionshoffnung bereit, wie seine Briefe an Bismarck selbst nach Ausbruch des Dänischen Krieges noch beweisen. So blieb der A.d.A.V. immer auch Stimmrechtsarmee. Weil Moses Hess in Lassalle den künftigen Diktator einer siegreichen Revolution erkannte, so mochte er soziale Thesen verbreiten, die von der Assoziationsidee unabhängig waren. Auf ein ähnliches Angebot von Rodbertus hat er nicht reagiert. ${ }^{2}$

Lassalle wollte in seinem Verein nicht soziale Aufklärung, sondern Indoktrinierung. "Nachbeten” hat Hess es später genannt. ${ }^{3}$ Deshalb war dort kein Platz für Rodbertus, sondern nur für seinen Namen. In dessen sozialökonomischem Erziehungsprogramm, ${ }^{4}$ das er in seinen Briefen angedeutet hatte, war kein Punkt, mit dem Lassalle nicht einverstanden sein konnte. Aber mit dieser Aufklärung war ein grosses Risiko verbunden: Die Schwächen des Assoziationsprogramms, die Lassalle im Briefwechsel zugeben musste, wären in den Reihen der Arbeiter zur Sprache gekommen, und das ging nicht an.

\section{IX}

Die Ausschaltung Rodbertus' von positivem Wirken im A.d.A.V. spiegelt sich im dritten Teil des Briefwechsels wider. Das Haupt-

INachgel. Briefe, Bd. 6, S. 339. Nr. Is.

2 Moses Hess bezeugt im „Nordstern" die Notwendigkeit einer persönlichen Diktatur, um den sozialen Neuerungen Bahn zu brechen (5.XII.1863). In Nr. 255 nennt er Lassalle den kommenden Diktator. Dann gehörte Hess dem Brüderbunde aller Kommunisten an, zu dem weder Ziegler noch Rodbertus gehörten. Daher Liebknechts Sonderstellung in Berlin.

${ }^{3}$ Hess an J. P. Becker (10.VIII.1 868).

4 Nachgel. Briefe, Bd. 6., S. 333. Nr. II. 
interesse gilt nunmehr der theoretischen Diskussion, bei der Rodbertus' Bodenrententheorie die Hauptrolle spielt. Ausgangspunkt ist das Problem des sozialen Ausgleichs in einer von Produktivassoziationen beherrschten Gesellschaft, denn die differentiale Bodenrente ist eine der Hauptursachen der sich erneuernden sozialen Ungleichheit. Aber selbst in dieser sich immer mehr von praktischen Gesichtspunkten entfernenden Diskussion versucht Rodbertus in Lassalle den geistigen Erben zu finden. Die Tatsache, dass Lassalle die ökonomischen Fragen zum ersten Mal mit Erfolg in die Öffentlichkeit getragen hat, ist für ihn ausschlaggebend, nachdem das ihm selbst in jahrzehntelanger Anstrengung nicht geglückt ist. Wie für Ziegler, so kann Lassalle auch für ihn der "Ultor" werden. ${ }^{1}$ Deswegen - und nicht aus Rechthaberei - ,zerniert" er Lassalle und will ihn in dieser so ganz theoretischen Frage zur Kapitulation zwingen. Aber zu einer wirklichen Auseinandersetzung kann es nicht kommen, weil Lassalle auch hier an die Fragen scholastisch herangeht. Er zitiert, was er oder Ricardo gesagt oder nicht gesagt haben, wie er es schon vorher mit der Erörterung des „Systems” gehalten hat. ${ }^{2}$ Er kann über Ricardo nicht hinaus, so wie er nicht über das Vorbild der Französischen Revolution hinaus kann. Er ist früh vollendet; er ist stecken geblieben.

Lassalle ist unvorbereitet an die Arbeiteragitation gegangen, aber nicht, wie er es den Leipzigern darstellte, aus Zeitmangel, sondern, wie uns der Fortgang seiner „Selbstverständigung” auch im Briefwechsel mit Rodbertus beweist, weil er nicht weiter konnte. Den Grundproblemen, die ihm Rodbertus stellte, ist er zur Zeit des „BastiatSchulze" ebenso ausgewichen wie zur Zeit des „Offenen Antwortschreibens". Den Beweis für sein „ehernes Lohngesetz" bildeten Zitate aus den verschiedensten nationalökonomischen Werken, darunter veraltete Wälzer. Die Mühe und den Zeitaufwand für solches anti-, quarisches Studium hat er nicht gescheut. Jahrelanges Ringen um eine „begriffliche” Nationalökonomie hat ihn dem Ziel nicht näher gebracht, aber trotzdem irrt Rodbertus sehr, wenn er später gemeint hat, er würde schliesslich Lassalle überzeugt haben. Sein Lavieren in der Frage der Assoziationen hätte ihn anders belehren können, denn der „,neue Mittelstand" als Idealbild der kommunistischen Gesellschaft erscheint in der Antikritik zum "Bastiat-Schulze”, weil er in den

1S. 333. Das Bekenntnis Rodbertus' zur Volksagitation ist aufschlussreich, will nicht nur für ein paar Professoren schreiben. Er erhofft eine Art Arbeitsteilung: Lassalles Agitation und seine Aufklärung. „Und darum dreimal Glückauf zu Ihrer Agitation!” 2 S. 370-371. Die Argumentation lautet: Wenn er zugäbe, dass auf dem schlechtesten Boden noch Bodenrente gewonnen werden muss, so wäre Ricardo widerlegt; wo hätte er das aber zugegeben? 
Manuskriptbriefen der Jugendzeit vorgezeichnet ist; der Kampf gegen Stimmrechts- und Steuerprivileg erscheint in der Arbeiteragitation, weil er der Dynamik der Französischen Revolution nachgebildet scheint. Auf Rodbertus' Ceterum censeo der Gefahr des kommenden Cäsarismus antwortet er nur mit einem Stossseufzer und dem ,alten Glauben”, denn darüber sagt ihm die Theorie der Jugendzeit nichts!

Lassalles Hilflosigkeit gegenüber dem Problem des Cäsarismus, das doch das Hauptproblem der Schicksalsjahre seiner Arbeiteragitation war, beweist, wie unzeitgemäss und abstrakt seine Begriffe von Demokratie und Revolution im Laufe weniger Jahre schon geworden waren. Es liegt Ironie darin, dass Lassalle so oft als Realpolitiker angesehen wird, nur weil eine Bewegung seinen Namen trug, und er mit Bismarck Beziehungen unterhalten hat. Auch diese Gespräche sind in ihrem sozialpolitischen Teil genau so weit von Realpolitik entfernt wie die theoretischen Erörterungen mit Rodbertus.

Lassalles Zukunftsschau ist ebenso total wie die eines Rodbertus, und seine „Diktatur der Einsicht” ganz von ihr getragen. Bei dem Versuch der Eroberung der Arbeiterbewegung hat ihm die Beimischung des ungeklärten utopischen Elements dauernd geschadet. Man darf nicht nur auf die relativen Erfolge Lassalles blicken, sondern muss das selbstbewusste Fernbleiben des grösseren Teils der organisierten Arbeiterschaft beachten. Lassalles Bewegung war der bestorganisierte Splitter der deutschen Arbeiterbewegung, aber doch nur ein Splitter.

Die deutschen Arbeiter blieben Lassalles Bewegung aus liberaldemokratischer Überzeugung fern. Deshalb hätten sie auch das staatstotalitäre System eines Rodbertus nie und nimmer angenommen, da für sie die soziale Frage ein Problem der Emanzipation war und nicht nur Frage des ,suum cuique”. Wenn nun Rodbertus bei Lassalle den Ausgangspunkt von Individualismus und Liberalismus nachgewiesen hat, so hat er ihn ganz ungewollt auf die Verbindung zu Männern wie F. A. Lange oder L. Büchner verwiesen, für die Sozialismus verwirklichte Demokratie war. Hätte die Auseinandersetzung mit Rodbertus, in der Lassalle sich gegenüber Rodbertus soviel Blössen wegen der Beimischung individualistischer Elemente gab, ihn zu Weiterdenken gebracht, so hätte er zwar nicht Rodbertus aus seiner Isolierung befreit, sich selber aber in einen breiteren Strom gestellt.

Die Geschlossenheit und Logik von Rodbertus' Denken waren eine entscheidende Hindernis für dessen Wirken; die Widersprüche in Lassalles Denken aber eine grosse Chance für Lassalle, die historisch sich aber nicht verwirklicht hat.

Aus vorgefasster Schau heraus hat Rodbertus den kommenden 
Cäsarismus in Deutschland nur als soziale Diktatur gesehen. Deshalb hat er ihn sowohl gefürchtet wie erwartet. Der Cäsarismus des ökonomischen "laissez faire" hat ihn überrascht und enttäuscht. Lassalle dagegen hat aus vorgefasster Schau nur gegenseitiges Aufreiben von Reaktion und Liberalismus in Rechnung gezogen. Für den Fall des Sieges eines manchesterlichen Cäsarismus, der der kapitalistischen Entwicklung die Wege ebnen wollte, hat er seiner Bewegung keinerlei gedankliche oder taktische Waffen in die Hand gegeben.

Der historische Weg für eine reale soziale und demokratische Arbeiterpolitik hätte sich vielleicht aus einer öffentlich geführten Auseinandersetzung der totalen Systeme von Rodbertus und Lassalle ergeben können. Dazu ist es aber nicht gekommen. 